\section{P-247 FROM MANAGING TO LEADING - UNLOCKING THE HIDDEN TALENT}

Robert Standfield. St. Richard's Hopsice, Worcester, UK

10.1136/bmjspcare-2016-001245.268

One of the key operating principles from the working paper of the Commission into the Future of Hospice Care (2012) identified leadership as a core requirement both at the strategic and operational level within organisations. St. Richard's Hospice has been fortunate in that two members of the senior management team have completed the MA in Hospice Leadership at Lancaster University. We recognised that to embrace the new era of empowering the wider teams and individuals to recognise leadership as a core skill and understand how they can influence current and future development of the sector is core to sustainability.

Up until recently most of the investment in leadership and management has been directed toward individuals where there was an element of self-selection linked to career aspirations or performance management issues where new skills and knowledge were required to deliver their role.

The hospice has now developed and implemented a process where the identification of leadership and management is a bottom-up approach starting at the annual appraisal linked to identifying and nurturing future leaders. We recognised that many of our current staff did not recognise that they already possess inherent skills especially in terms of emotional intelligence, innovation and personal motivation.

As a hospice we have:

- Provided people with the knowledge about how to make best use of appraisal and demonstrating the impact in organisational development.

- Emphasised the hospice strategic objectives, linking their current role to how they are delivering against these objectives and empowering them to contribute to our future strategic direction.

- Establishing longer term career aspirations and navigating people toward achieving these.

- Creating opportunities for staff to complete formal leadership and management qualifications (ILM Levels 3 and 5).

The next stage in the process, identified by the future leaders is establishing the need for coaching and mentoring and how this will support continuous improvement.

\section{P-248 HARMONISING THE WORKING STRUCTURE OF AN IN- PATIENT UNIT EMBEDDING A CULTURE SHIFT}

Jacquie Pamphilon, Karen Causton. St Helena Hospice, Colchester, UK

\subsection{6/bmjspcare-2016-001245.269}

Introduction Hospice nurse leaders need to promote a safe effective environment for patients requiring end-of-life care, whilst being a mentor for less experienced palliative nurses. The hospice inpatient unit manager observed a disparity in roles when registered nurses work as shift leaders. Some shift leaders have not had leadership skills training and do not always have the skills to create process and offer clear direction. Nurse leaders need to achieve good outcomes for all not just a few, to achieve this they need to reflect on and question leadership and management practices (Ellis and Bach 2015)
Aim and objectives

- Coaching RNs to understand leadership practices as shift leaders and the importance of their role in influencing the development of new nurses

- Encourage a culture change for shift leaders to realise the potential of being stong leaders focusing on patient wellbeing.

Approach used - work in progress The unit manager and learning and development officer working in partnership on a joint project investigated reasons for the disparity. Shift leaders spent time reflecting on their approach to leadership, outcomes provided a base of their then situation. Observation allowed for deeper understanding of the nurse's practice (Keatinge 2002). Discussion of the observations included; could understanding and implementing leadership styles harmonise the team, reflection on management theories such as Belbin's team roles (Belbin 2004)

Evaluation RNs recognising their responsibilities as leaders and seeing a growth in project work and knowledge sharing that will educate new nurses.

Conclusion This culture shift is important to bridge gaps in leadership as effective leadership is critical in delivering high quality care and ensuring patient safety and facilitating positive staff development (Frankel 2008). Sharing an approach that looks at leadership styles and what they mean on IPU might offer alternative methods for solutions in other hospices.

\section{P-249 THE ROLE OF HOSPICES IN THE EMERGING WORLD OF INTEGRATED AND DEVOLVED HEALTH AND CARE SYSTEMS}

1,2,3,4,5Steve Dewar, 1,4,5,6,7,8 Judy Taylor, 1,7,8John Harries, 1,5,6,7 Janet Wilson, ${ }_{1,4,7,8}$ Amanda Wilsher. ${ }^{1}$ The Westcott Group Ltd; ${ }^{2}$ Greenwich and Bexley Community Hospice; ${ }^{3}$ St Christopher's Hospice; ${ }^{4}$ Together for Short Lives; ${ }^{5}$ Hospice UK; ${ }^{6}$ NHS Leadership Academy; ${ }^{7}$ Leadership Development, King's Fund; ${ }^{8}$ University of Birmingham

\subsection{6/bmjspcare-2016-001245.270}

Background Strengthening the leadership of hospice care is one of five key steps identified by the Commission into the Future of Hospice Care to ensure that hospices are fit for the future.

The landscape for hospice leaders is complex and challenging. The majority of hospices have seen their statutory funding remain static or even fall. The demand for end-of-life care is predicted to increase significantly and NHS and local authorities are urging local leaders to develop end of life care systems that are integrated, accessible, systematic and responsive.

To meet these challenges, hospice leaders need a coherent story about the role of their organisations in the future, their capacity to contribute to new systems of care and their ability to lead complex change.

Hospice leaders on the Hospice UK Strategic Leadership Programme will be identifying key features of this future landscape for hospice care. The 2016 Conference provides an exciting opportunity to share their emerging vision for the future with the wider hospice community.

Aims Hospice leaders on the Strategic Leadership Programme will be exploring the national landscape of end-of-life care and shaping their own response to the challenges. The aim is to bring these responses to the challenges to the 2016 conference, to stimulate debate and inspire leadership across the sector.

Methods During the first two modules of the Strategic Leadership Programme a cohort of over 30 hospice leaders from more than 20 hospices will develop an understanding of the potential and 